\title{
Citodiagnóstico en otorrinolaringología. Diez años de experiencia en el Hospital Barros Luco Trudeau 2001 a 2010
}

\author{
Citodiagnostic in Otorhinolaryngology. Ten years of experience \\ in Barros Luco Hospital 2001 to 2010
}

Pilar Gajardo 01, Orlando Quezada P², Catalina Gutiérrez C³, Maritza Rahal E².

\begin{abstract}
RESUMEN
Introducción: Dentro del estudio diagnóstico de pacientes con masa cervical la punción por aspiración con aguja fina (PAAF) es una herramienta útil debido a su facilidad y seguridad.

Objetivo: Evaluar el rendimiento de las PAAF realizadas en nuestro servicio en los últimos diez años.

Material y método: Estudio retrospectivo y descriptivo. Se obtuvieron los datos de pacientes sometidos a PAAF en el Servicio de Otorrinolaringología del Hospital Barros Luco Trudeau entre los años 2001 a 2010 y se correlacionaron con las biopsias definitivas atingentes.

Resultados: Se realizaron 197 PAAF, de las cuales 140 (71\%) fueron aptas para estudio citológico y 77 tenían biopsias concomitantes. Se obtuvo una sensibilidad de $46 \%$, especificidad de $85 \%$, valor predictivo positivo de $74 \%$ y valor predictivo negativo de $62 \%$. La localización para la cual se obtuvo un mejor rendimiento de la técnica fue para glándulas salivales y los diagnósticos con mejor rendimiento fueron carcinoma escamoso y tumor mixto.

Discusión: La PAAFes una herramienta subutilizada. Obtuvimos una alta tasa de muestras insuficientes para estudio diagnóstico y, en nuestro medio, es útil para descartar neoplasia maligna en masas de glándulas salivales y metástasis de carcinoma escamoso.
\end{abstract}

Palabras clave: Masa cervical, punción por aspiración con aguja fina, citología.

\begin{abstract}
Introduction: Fine needle aspiration (FNA) is an useful tool for the diagnostic evaluation of patients with a cervical mass, because of its feasibility and safety.

Aim: To determine the accuracy of FNA cytology in our hospital over the last ten years.

Material and method: A retrospective and descriptive study. Data were obtained from patients undergoing FNA in the Department of Otolaryngology, Hospital Barros Luco Trudeau from 2001 to 2010 and compared with the corresponding histology report of the original surgical specimen.
\end{abstract}

Servicio de Otorrinolaringología, Hospital Barros Luco Trudeau.

Servicio de Anatomía Patológica, Hospital Barros Luco Trudeau.

Médico General. 
Results: A total of 197 FNA procedures were performed in 143 patients. A definite cytological diagnosis was made in 140 patients (71\%). 77 patients had corresponding histology samples. The sensitivity was $46 \%$, specificity $85 \%$, positive predictive value $74 \%$ and negative predictive value $62 \%$. The location for which we obtained a better performance of the technique was in salivary glands and the best performing diagnosis was squamous cell carcinoma.

Discussion: FNA is an underused diagnostic tool with a high rate of non diagnostic samples. It is useful to rule out malignancy in salivary gland masses and metastatic squamous cell carcinoma in lymph nodes.

Key words: Cervical mass, fine needle aspiration, cytology.

\section{INTRODUCCIÓN}

Dentro de los motivos de consulta a los que un otorrinolaringólogo debe enfrentarse en su práctica clínica habitual se encuentra el paciente con una masa cervical. En el estudio diagnóstico de este paciente, la punción por aspiración con aguja fina (PAAF) es una herramienta muy útil debido a que es un procedimiento que se realiza ambulatoriamente, es bien tolerado por el paciente, tiene un bajo costo y la interpretación por el citopatólogo es rápidaa, ${ }^{1,2}$. Las masas cervicales se localizan, generalmente, en planos superficiales, siendo palpables y de fácil acceso para puncionar ${ }^{2}$. Dentro de las desventajas de esta técnica se describe que quien toma la muestra y quien la interpreta deben tener un entrenamiento adecuado, especialmente importante en el caso del citopatólogo, quien para poder tener la experiencia suficiente, debe enfrentarse cotidianamente a muestras citológicas de cabeza y cuello. No existen contraindicaciones para la realización de este procedimiento y sus complicaciones son poco frecuentes, tales como el sangrado o la formación de un hematoma en el sitio de punción ${ }^{3}$.

Un reciente metaanálisis que incluyó 3.459 aspirados de masas de cabeza y cuello mostró para esta técnica diagnóstica una sensibilidad de $89,6 \%$, especificidad de $96,5 \%$ y una exactitud diagnóstica de $93,1 \%$. El rendimiento de la PAAF varió según localización de la masa cervical y según diagnóstico histológico, siendo las lesiones ubicadas en ganglios cervicales y los adenocarcinoma y carcinoma escamoso, respectivamente, aquellas en las cuales el estudio citológico tendría un mejor rendimiento ${ }^{4}$. Cabe destacar que los estudios muestran que el valor diagnóstico de la PAAF mejora cuando ésta es realizada por grupos de clínicos y citopatólogos de amplia experiencia que trabajan en equipo $0^{5,6}$.

\section{OBJETIVO}

El objetivo de nuestro estudio es evaluar el rendimiento de las PAAF realizadas en nuestro servicio en los últimos diez años y compararlo con lo descrito a nivel nacional e internacional.

\section{MATERIAL Y MÉTODO}

Se realizó un estudio descriptivo retrospectivo. A partir de la base de datos del Servicio de Anatomía Patológica del Hospital Barros Luco Trudeau se obtuvieron los datos de los pacientes sometidos a PAAF en el Servicio de Otorrinolaringología, en el contexto del estudio de una masa cervical, entre enero de 2001 a diciembre de 2010. Posteriormente se obtuvieron las biopsias definitivas concordantes anatómica y temporalmente (dentro de doce meses de tomada la PAAF) de los pacientes seleccionados, las cuales fueron consideradas como el patrón de oro del diagnóstico histológico. Se excluyeron todas las punciones tiroideas, ya que en nuestro hospital dicho procedimiento es llevado a cabo por médicos no otorrinolaringólogos.

Todas las PAAF fueron realizadas por médi$\cos$ de nuestro servicio, ya sea residentes 0 especialistas, según la técnica descrita por Amedee $^{3}$, sin ecografía ni guía radiológica alguna, y analizadas por el mismo citopatólogo del Servicio de Anatomía Patológica del Hospital Barros Luco Trudeau.

Las variables a analizar fueron características sociodemográficas de los pacientes, el tiempo de evolución de la masa cervical, la localización de ésta, presencia de estudio imagenológico complementario y estudio citológico e histológico de la masa. 
Como medida resumen se utilizó el promedio para los datos que distribuían en forma normal. Se hicieron los cálculos de especificidad, sensibilidad, valor predictivo positivo (VPP) y valor predictivo negativo (VPN) para el diagnóstico de neoplasia con los resultados del estudio citológico de las muestras obtenidas mediante PAAF y los resultados de la biopsia definitiva.

\section{RESULTADOS}

Durante el período estudiado se realizaron en nuestro servicio ciento noventa y siete PAAF en ciento cincuenta y tres pacientes, de los cuales el $58 \%$ correspondían a pacientes de género masculino con una edad promedio de cincuenta y tres años. El promedio de PAAF en nuestro servicio fue de veinte punciones por año.

\section{CARACTERÍSTICAS CLÍNICAS}

De los ciento cincuenta y tres pacientes sometidos a PAAF, el tiempo promedio de evolución de su masa cervical al momento del diagnóstico fue de quince meses. El $70 \%$ de los pacientes contaba con estudio imagenológico complementario. De aquellos con estudio imagenológico, 56\% presentaba tomografía computarizada (TC) de cuello, 33\% presentaba TC de cuello y ecografía cervical y finalmente $11 \%$ presentaba solamente ecografía cervical.

Con respecto a la localización de la muestra, $25 \%$ de las PAAF no presentaban la información respecto del sitio de toma de muestra. De aquellas que informaban la localización, 46\% correspondían a muestras obtenidas de glándula salival mayor, $27 \%$ fue obtenida de ganglio cervical y $27 \%$ se obtuvo de otras localizaciones tales como fosa nasal y paladar. Dentro de las muestras obtenidas de glándula salival mayor, $72 \%$ correspondía a muestras obtenidas de glándula parótida, $26 \%$ de glándula submaxilar y $2 \%$ de glándula sublingual.

\section{RESULTADOS CITOLÓGICOS}

De las ciento noventa y siete PAAF evaluadas, $56(28 \%)$ fueron informadas como muestra inadecuada para diagnóstico citológico. Ciento once $(56 \%)$ fueron informadas como negativas para células neoplásicas, 27 (14\%) positivas para células neoplásicas, 2 muestras fueron interpretadas como sospechosas para células neoplásicas y finalmente una muestra no presentaba informe citológico. De aquellas muestras informadas como negativas para células neoplásicas, los diagnósticos citológicos más frecuentes fueron exudado inflamatorio (34\%), adenoma pleomorfo (tumor mixto) $(23 \%)$ e hiperplasia ganglionar (15\%) (Figura 1). En las muestras positivas para células neoplásicas, los diagnósticos citológicos más frecuentes fueron carcinoma escamoso $(47 \%)$, linfoma no Hodgkin (11\%) y adenocarcinoma papilar $(7 \%)$. Dentro de las muestras positivas para células neoplásicas hubo $11 \%$ en que no se especificó el tipo de neoplasia (Figura 2). Las causas más frecuentes de muestra inadecuada para estudio citológico fueron muestra acelular $(58 \%)$ y muestra hemorrágica (30\%) (Figura 3).

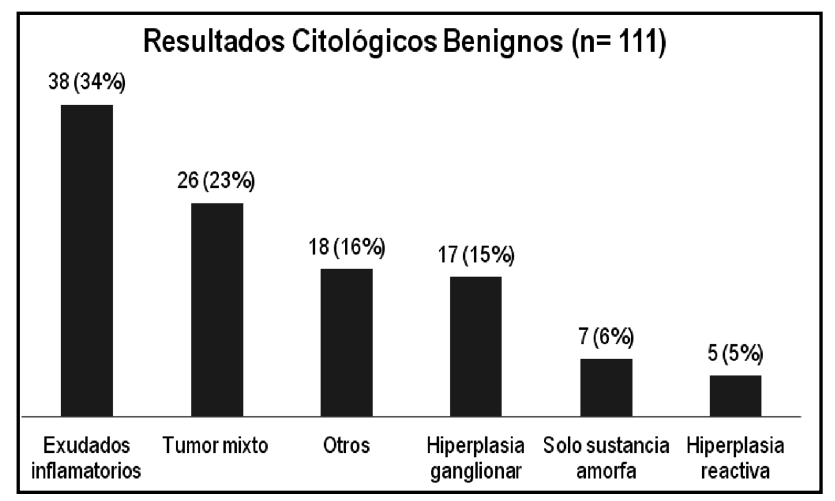

Figura 1. Diagnósticos citológicos benignos. 


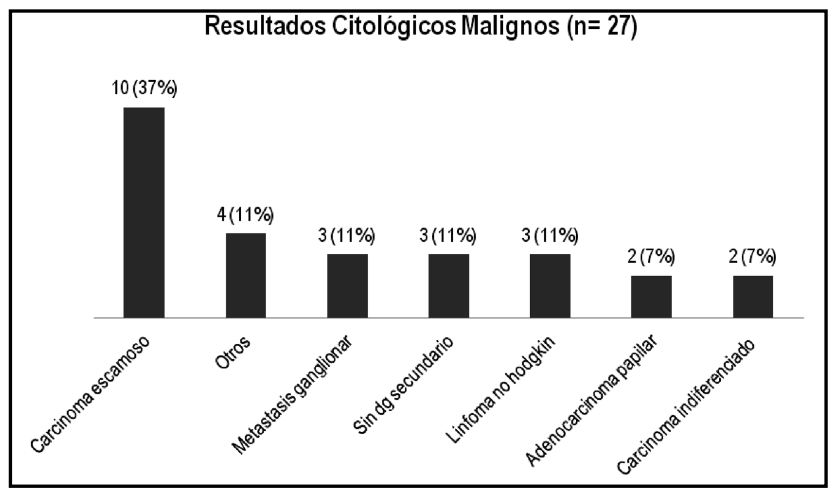

Figura 2. Diagnósticos citológicos positivos para neoplasia.

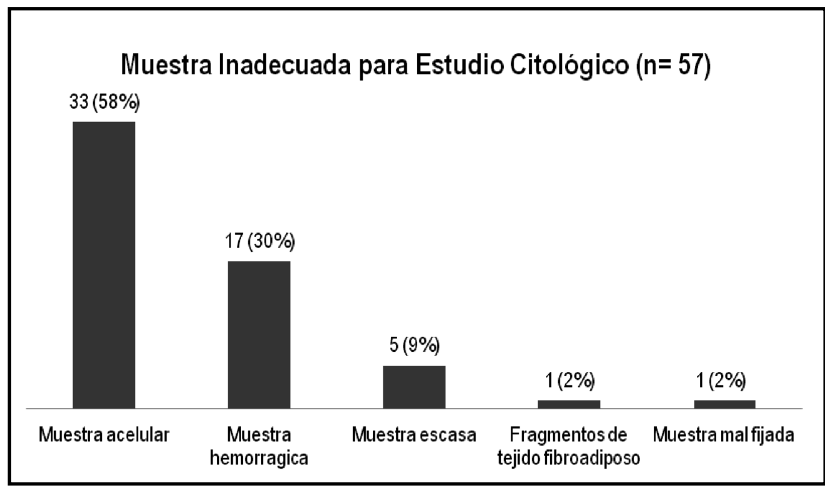

Figura 3. Causas de muestras inadecuadas para estudio citológico.

\section{Rendimiento diagnóstico de la PAAF}

Del total de PAAF con diagnóstico citológico (ciento cuarenta), solamente setenta y siete (55\%) contaban con muestras histológicas atingentes disponibles, las cuales fueron finalmente analizadas para determinar el rendimiento diagnóstico de la PAAF en nuestro servicio. En la Tabla 1 se observa la tabla de contingencia de nuestro estudio, a partir de la cual se realizaron los cálculos estadísticos. Para el diagnóstico de neoplasia mediante estudio citológico de muestras obtenidas mediante PAAF se obtuvo una sensibilidad de $46 \%$, una especificidad de $85 \%$, un VPP de $74 \%$, un VPN de $62 \%$.

Al analizar el rendimiento de la PAAF según localización de la muestra, obtuvimos que la sensibilidad y VPP para neoplasia es mayor en muestras tomadas de ganglio cervical versus aquellas tomadas de glándulas salivales. Sin embargo, en esta última localización, la especificidad y el VPN para neoplasia son mejores (Tabla 2).
Al evaluar nuestros resultados según histología, obtuvimos que el diagnóstico histológico benigno y maligno para el cual el estudio citológico tiene mejor rendimiento fue para adenoma pleomorfo y carcinoma escamoso, respectivamente (Tabla 3).

\section{Rendimiento diagnóstico de la repetición de la PAAF}

Una de las dudas frecuentes frente a la obtención de muestras para estudio citológico es si repetir la toma de muestra mejora el rendimiento de esta técnica diagnóstica. En nuestro estudio, en aquellos pacientes sometidos a una toma de muestra hubo una concordancia citológica-histológica (benignidad-benignidad 0 malignidad-malignidad, sin especificar diagnóstico específico) en el $70 \%$ de los casos, con una exactitud diagnóstica (igual diagnóstico específico) en el $62 \%$ de los casos. En los pacientes que fueron sometidos a dos tomas de 
Tabla 1. Tabla de contingencia para evaluar el rendimiento diagnóstico de la punción por aspiración con aguja fina para el diagnóstico de neoplasia

\begin{tabular}{|lcccc|}
\hline \multicolumn{4}{c|}{ Biopsia definitiva } \\
\hline PAAF & Histología maligna & Histología benigna & TOTAL \\
\hline & Citología maligna & 17 & 6 & 23 \\
& Citología benigna & 20 & 34 & 54 \\
\hline TOTAL & 37 & 40 & 77 \\
\hline
\end{tabular}

Tabla 2. Rendimiento diagnóstico de la punción por aspiración con aguja fina según localización de la masa cervical

\begin{tabular}{|lccccc|}
\hline Localización & N & Sensibilidad & Especificidad & VPP & VPN \\
\hline Glándulas salivales & 36 & $30 \%$ & $89 \%$ & $50 \%$ & $77 \%$ \\
Ganglio cervical & 19 & $50 \%$ & $80 \%$ & $88 \%$ & $36 \%$ \\
Otros & 22 & $54 \%$ & $78 \%$ & $78 \%$ & $54 \%$ \\
\hline
\end{tabular}

Tabla 3. Rendimiento diagnóstico de la punción por aspiración con aguja fina según diagnóstico histológico

\begin{tabular}{|lccccc|}
\hline Diagnóstico & N & Sensibilidad & Especificidad & VPP & VPN \\
\hline Tumor mixto & 18 & $83 \%$ & $78 \%$ & $79 \%$ & $82 \%$ \\
Carcinoma escamoso & 14 & $53 \%$ & $90 \%$ & $57 \%$ & $89 \%$ \\
Carcinoma de glándulas salivales & 7 & $29 \%$ & $90 \%$ & $40 \%$ & $84 \%$ \\
Linfoma & 6 & $17 \%$ & $99 \%$ & $50 \%$ & $92 \%$ \\
\hline
\end{tabular}

muestra, la concordancia citológica-histológica fue de $75 \%$ y la exactitud diagnóstica de $50 \%$. Finalmente en aquellos sujetos sometidos a tres tomas de muestra, la concordancia citológica-histológica fue de $43 \%$, con una exactitud diagnóstica de $0 \%$ (Figura 4).

\section{DISCUSIÓN}

Durante los 10 años correspondientes al período de estudio, se realizaron en el Servicio de Otorrinolaringología del Hospital Barros Luco Trudeau en promedio veinte PAAF de masas

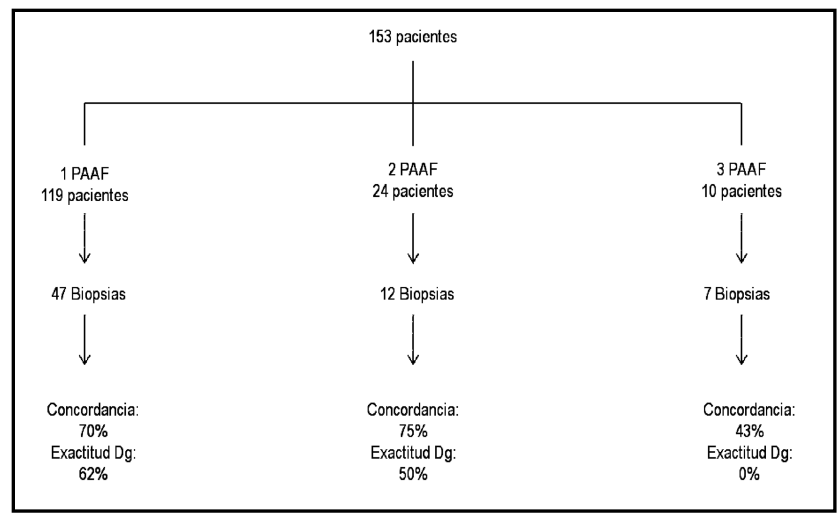

Figura 4. Rendimiento diagnóstico de la paaf al repetir la toma de muestra. 
cervicales anuales, número que nos parece bajo, debido al alto número de pacientes nuevos vistos en nuestro servicio (aproximadamente siete mil pacientes nuevos al año). Lo anterior, sumado a que solamente el $65 \%$ de las parotidectomías realizadas en el mismo periodo de tiempo contaban con un estudio citológico previo a la cirugía, nos hace plantear que en la actualidad el estudio citológico de las masas cervicales en una herramienta subutilizada en nuestro medio.

Llama la atención el alto porcentaje de muestras insuficientes para estudio citológico obtenidas en nuestro estudio (28\%), lo cual está dentro de rango de lo reportado en la literatura internacional, donde se describe desde $3 \%$ al $52 \%$ de muestras no diagnósticas $2,4,7$. En el trabajo de Wu y cols se describe que el $33 \%$ de las punciones tomadas por clínicos fueron inadecuadas para estudio citológico, lo cual es similar a lo reportado en nuestro trabajo. Sin embargo, de las punciones obtenidas por patólogos, solamente $7 \%$ fueron inadecuadas para estudio citológico, diferencia que los autores atribuyen a que los citopatólogos tendrían mejor entrenamiento que los clínicos en obtener muestras para esta técnica diagnóstica. En nuestro caso, creemos que nuestros resultados pueden deberse al escaso número de punciones realizadas en este período de tiempo, lo cual pudiese estar influyendo en que los médicos en formación no estén adquiriendo las destrezas necesarias para este procedimiento.

El rendimiento diagnóstico de la PAAF para el estudio de masas cervicales en esta casuística es inferior a lo reportado en literatura anglosajona ${ }^{4}$ y a lo reportado en nuestro centro el año $1996^{8}$, donde del análisis de veinte y un muestras de pacientes con características clínicas y demográficas similares a los pacientes de nuestro estudio, se obtuvo una sensibilidad de $85,7 \%$ y una especificidad de $100 \%$ para el diagnóstico de neoplasia. Con los resultados actuales postulamos que la PAAF de masas cervicales tomada por otorrinolaringólogos de nuestro servicio tendría un rol en descartar lesiones neoplásicas malignas de glándulas salivales dada su alta especificidad (89\%) y VPN $(77 \%)$. Es decir, en los pacientes con aumento de volumen de glándula salival mayor, en que la PAAF arroja un resultado negativo para células neoplásicas, 9 de cada 10 de ellos tendrá realmente una masa benigna. Según los resultados obtenidos, en nuestros pacientes, la PAAF sería una buena herramienta para descartar metástasis ganglionar de carcinoma escamoso, tipo histológico más frecuente de las neoplasias de vía aerodigestiva superior, ya que para este diagnóstico histológico se obtuvo una especificidad de $90 \%$ y VPN de $89 \%$. Para el diagnóstico histológico de linfoma, nuestra muestra es pequeña para interpretar los resultados con exactitud (tres casos). Sin embargo, la literatura reporta que es particularmente difícil poder diferenciar con citología entre un ganglio reactivo con hiperplasia linfoide y un linfoma de bajo grado².

Repetir la obtención de la muestra citológica no mejoraría el rendimiento diagnóstico, sino que haría más probable la obtención de una muestra suficiente para estudio, por lo cual en nuestro medio, sugerimos repetir la PAAF si la primera muestra fue inadecuada para estudio citológico. Este resultado es similar a lo descrito por Brennan ${ }^{9}$ y Shykhon ${ }^{10}$, quienes en estudios diferentes, plantean que la segunda PAAF debería ser tomada en los casos en que la primera muestra fue insuficiente para estudio citológico.

El $15 \%$ de falsos negativos debe ser interpretado con cautela e idealmente se debe analizar si esto se debe a un error de la muestra 0 a un error del análisis citológico, con el fin de mejorar nuestras deficiencias actuales, para mejorar el rendimiento de esta técnica a futuro en nuestro centro asistencial. Los valores obtenidos deben ser analizados con cautela, ya que debemos recordar que aquellos pacientes para los cuales se obtuvo un resultado citológico benigno no tumoral, dentro de los cuales se encuentran la mayoría de las muestras de exudados inflamatorios, no fueron sometidos a cirugía, por lo cual no se obtuvo histología, no siendo incluidos en el cálculo del rendimiento diagnóstico de la PAAF. La especificidad y VPN de la PAAF en nuestro medio podrían ser mayores, porque lo más probable es que estos casos sean verdaderos negativos (si tomamos la evolución clínica de estos pacientes que no presentaron malignidad en su seguimiento).

En la actualidad existen una serie de complementos que ayudan a optimizar el rendimiento diagnóstico de la PAAF, desde mejorar la obtención de una muestra, hasta técnicas moleculares que lograrían la obtención de un diagnóstico citológico 
más certero. Con respecto a la obtención de una muestra adecuada, tema muy relevante según lo comentado en párrafos anteriores, en que diferentes estudios describen tasas de muestras inadecuadas altas, la toma de PAAF bajo visión ecográfica es una opción útil en la actualidad, sin embargo, existen resultados controversiales si esta técnica imagenológica ayudaría a obtener un mejor porcentaje de muestras adecuadas para estudio citológico (excluyendo punciones tiroideas) ${ }^{2,11}$. Otra alternativa enfocada a mejorar el rendimiento de esta técnica es la lectura inmediata de la muestra por el citopatólogo, lo cual ayudaría a obtener mejores resultados por la calidad de la muestra y mejoraría los tiempos al diagnóstico, ya que en caso de obtener una muestra insuficiente para estudio, esto sería detectado inmediatamente, y el paciente ser sometido en el mismo momento a una segunda PAAF ${ }^{12}$.

En los últimos años se han aplicado a muestras citológicas tinciones de inmunohistoquímica y moleculares que sumarían beneficios a esta técnica diagnóstica. Con respecto a muestras obtenidas de cabeza y cuello, se ha demostrado recientemente que es posible detectar la presencia de virus papiloma humano mediante inmunohistoquímica, realizar estudios de genotipo y detectar proteínas supresoras de tumores tales como p16, en muestras obtenidas mediante PAAF $^{13,14}$.

Es importante señalar que el estudio citológico es una orientación diagnóstica y no corresponde al diagnóstico definitivo. Junto con la clínica del paciente, la PAAF ayuda a orientar acerca de la etiología de una masa cervical y así la conducta a seguir, antes de obtener el diagnóstico histológico final.

\section{CONCLUSIÓN}

Si bien nuestros resultados muestran que la PAAF es una buena herramienta diagnóstica para el descarte de enfermedad maligna en masas cervicales, debemos mejorar nuestra toma de muestra y nuestro análisis citológico, para poder utilizarla confiablemente en el estudio diagnóstico de un paciente con masa cervical. Es indispensable tener una fluida comunicación entre clínicos y citopatólogos, y estar pendiente de formar a las nuevas generaciones en la adecuada técnica del procedimiento y análisis de especímenes citológicos, con el fin de mejorar constantemente esta herramienta diagnóstica.

\section{Agradecimientos}

Agradecemos al equipo humano del Servicio de Anatomía Patológica del Hospital Barros Luco Trudeau por su colaboración en la obtención de la base de datos que hizo posible la realización de este estudio.

\section{BIBLIOGRAFÍA}

1. Wu M, Burstein DE, Yuan S, Nurse LA, Szporn AH, Zhang D, et AL. A comparative study of 200 fine needle aspiration biopsies performed by clinicians and cytopathologists. Laryngoscope 2006; 116: 1212-5.

2. Howlett DC, Harper B, Quante M, Berresford A, Morley M, Grant J, et al. Diagnostic adequacy and accuracy of fine needle aspiration cytology in neck lump assessment: results from a regional cancer network over a one year period. $J$ Laryngol Otol 2007; 121: 571-9.

3. Amedee RG, Dhurandhar NR. Fine-needle aspiration biopsy. Laryngoscope 2001; 111 : 1551-7.

4. Tandon S, Shahab R, Benton Jl, Ghosh SK, Sheard $J$, JonEs TM. Fine-needle aspiration cytology in a regional head and neck cancer center: comparison with a systematic review and metaanalysis. Head Neck 2008; 30: 1246-52.

5. Schwarz R, Chan NH, MacFarlane JK. Fine needle aspiration cytology in the evaluation of head and neck masses. Am J Surg 1990; 159: 482-5.

6. Wu M, Burstein DE. Fine needle aspiration. Cancer Invest 2004; 22: 620-8.

7. Zbaren P, Schar C, Hotz MA, Loosli H. Value of fine-needle aspiration cytology of parotid gland masses. Laryngoscope 2001; 111: 1989-92.

8. Bonilla G, Calabrese R, Quezada O. Punción Citológica en Masas de Cabeza y Cuello. Rev Otorrinolaringol Cir Cabeza Cuello 1997; 57: 39-42.

9. Brennan PA, Davies B, Poller D, Mead Z, Bayne D, Puxeddu R, et al. Fine needle aspiration cytology (FNAC) of salivary gland tumours: repeat 
aspiration provides further information in cases with an unclear initial cytological diagnosis. $\mathrm{Br} \mathrm{J}$ Oral Maxillofac Surg 2010; 48: 26-9.

10. Shykhon M, Macnamara M, El-Assy A, Warfield AT. Role of repeat fine needle aspiration cytology in head and neck lesions: preliminary study. $J$ Laryngol Otol 2004; 118: 294-8.

11. Carr S, Visvanathan V, Hossain T, Uppal S, Chengot P, WOODHEAD CJ. How good are we at fine needle aspiration cytology? J Laryngol Otol 2010; 124 : 765-6.

12. Moberly aC, Vural e, nahas B, Bergeson tR, KoKoska MS. Ultrasound-guided needle aspiration: impact of immediate cytologic review. Laryngoscope 2010; 120: 1979-84.

13. Solomides CC, BibBo M, Wang ZX. Assessment of fine needle aspiration specimen adequacy for high-risk HPV detection and genotyping in oropharyngeal squamous cell carcinoma. Acta Cytol 2012; 56: 196-8.

14. Jakscha J, Zlobec I, Storck C, Obermann EC, Tornillo L, Terracciano LM, et al. The clinical impact of p16 status in fine-needle aspirates of cervical lymph node metastasis of head and neck squamous cell carcinomas. Eur Arch Otorhinolaryngol 2012. 